\title{
Manifestation of Heterosis in Bittergourd
}

\author{
P. Robindro Singh*, ChandanKarak, Priyadarshani P. Mohapatra, \\ B. Ashok Kumar and P. Hazra
}

Department of Vegetable Crops, Bidhan Chandra Krishi Vishwavidyalaya (BCKV), Mohanpur, West Bengal-741252, India

*Corresponding author

\begin{tabular}{|c|c|}
\hline & A B S T R A C T \\
\hline & $\begin{array}{l}\text { The present investigation on "Manifestation of Heterosis in Bitter gourd (Momordica } \\
\text { charantia L)" has been undertaken at Central Research Farm, Gayeshpur, Bidhan Chandra } \\
\text { Krishi Viswavidyalaya, Nadia, and West Bengal during autumn-winter season of 2015-16 } \\
\text { to2016- } 2017 \text { aimed to assemblage of diverse inbred lines and development of hybrids, } \\
\text { evaluation of the parental genotypes and hybrids through morphological characters, fruit }\end{array}$ \\
\hline $\begin{array}{l}\text { K e y w o r d s } \\
\text { Bittergourd, } \\
\text { Heterosis, Specific } \\
\text { crossing programme }\end{array}$ & $\begin{array}{l}\text { yield components and fruit quality characters, study on the inter-relationships among } \\
\text { different characters with a view to ideotype breeding for enhancing fruit yield and quality, } \\
\text { analysis of the manifestation of heterosis for different characters and identification of } \\
\text { promising hybrids suitable for this growing condition. For this purpose, seven diverse } \\
\text { parental lines viz., Kerala white, BG-1346501, Pusa Vishesh, Meghna-2, Itki, Special }\end{array}$ \\
\hline Article Info & Boulder and Murhu. And Development of 10 Hybrids by specific crossing programme. \\
\hline $\begin{array}{l}\text { Accepted: } \\
\text { 12 September } 2018 \\
\text { Available Online: } \\
10 \text { October } 2018\end{array}$ & $\begin{array}{l}\text { for which manifestation of heterosis was calculated. Negative heterosis, which is desirable } \\
\text { for days to appearance of first male and female flower, number of node bearing first male } \\
\text { and female flower and days to first fruit harvest, were common in most of the crosses. } \\
\text { Among } 10 \mathrm{~F}_{1} \text { hybrids, Meghna- } 2 \text { x BG- } 1346501 \text { and BG- } 1346501 \times \text { Special Boulder were }\end{array}$ \\
\hline & $\begin{array}{l}\text { recorded to be the two best performing } F_{1} \text { hybrids for fruit yield per plant. It is therefore, } \\
\text { suggested that these promising parents and crosses may be exploited for further } \\
\text { amelioration of yield and yield components in bitter gourd. The selected crosses can be } \\
\text { directly utilized as promising hybrids. }\end{array}$ \\
\hline
\end{tabular}

\section{Introduction}

Bitter gourd (Momordica charantia), one of the major cucurbitaceous vegetables of the family Cucurbitaceae is grown throughout India for its tender fruits. Bitter gourd has been used for centuries in the ancient traditional medicine of India, China, Africa, and Latin America as its extract possess antioxidant, antimicrobial, antiviral, antihepatotoxic, antiulcerogenic properties and also has the ability to lower blood sugar (Welthinda et al., 1986). During the past decade, the antidiabetic properties of the crop have been studied extensively and a hypoglycemic principle called "Charantin" has been isolated. The bitterness of bitter gourd is due to the cucurbitacin like alkaloid 
momordicine and triterpene glycosides viz., momordicoside K and L (Jeffrey, 1980 and Okabe et al., 1982).

Bitter gourd has been used in various herbal medicine systems for a long time because of its disease preventing and health promoting phyto-chemical compounds like dietary fiber, minerals, vitamins, flavonoids and antioxidants. It is also used for reduction of blood sugar levels in the treatment of type- 2 diabetes.

It is highly cross pollinated in nature due to monoecious sex form and has high levels of heterozygosity. Due to efforts of vegetable breeders, improved varieties and hybrids have been developed. Crop improvement involves strategies for enhancing yield potentiality and quality components. Selection of parents based on per se performance does not yield the desired results. Selection of parents for hybridization has to be based on the complete genetic information and prepotency of the potential parents. Heterosis breeding provides an opportunity for improvement in productivity, earliness, uniformity, quality and development of resistance to pests and diseases (Riggs 1988). With these points in view, heterosis and combining ability studies are prerequisite in any plant breeding programme, which provides the desired information regarding the varietal improvement or exploiting heterosis for commercial purposes. Though many reports on combining ability and heterosis breeding are available in bitter gourd (Sirohi and Choudhury, 1978; Lawande and Patil, 1990; Choudhari and Kale, 1991; Singh et al., 2013), information on identification of better parents for $F_{1}$ production is lacking.

\section{Materials and Methods}

The field experiments were carried out at Central Research Farm, Gayeshpur, Bidhan
Chandra Krishi Viswavidyalaya, Nadia, and West Bengal, situated at $22^{\circ} 57^{\prime} \mathrm{N}$ latitude and $88^{\circ} 20^{\prime} \mathrm{E}$ longitudes with average altitude of $9.75 \mathrm{~m}$ above the mean sea level (MSL) during autumn-winter season. Topographic situation of the experimental site comes under Gangetic new alluvial plains of West Bengal with sandy loam soil.

\section{Assemblage of plant materials for the study}

Seven diverse inbred lines (variety and breeding lines) of bitter gourd viz., Kerala White, Special Boulder, Meghna-2, BG1346501, Pusa Vishesh, Murhu and Itki maintained in the BARC, Govt. of India sponsored research project "Nuclearintervened molecular breeding and in vitro culture for increasing yield and phytomedicine production in bitter gourd (Momordica charantia)" at the Department of Vegetable crops, Bidhan Chandra Krishi Viswavidyalaya constituted the parental genotypes for the present investigation.

\section{Development of Hybrids by specific crossing programme}

The following 10 hybrids were developed by crossing the diverse parental inbred lines following conventional hybridization method as under:-Kerala White X Meghna-2,Meghna2 X BG-1346501, BG-1346501 x Special Boulder, Meghna-2 X Murhu, BG-1346501 x Pusa Vishesh, Pusa Vishesh x Meghna-2, Special Boulder x Kerala White, BG-1346501 x Itki, Pusa Vishesh x BG-1346501,BG$1346501 \mathrm{X}$ Murhu.In this hybridization method, the buds of the staminate and pistillate flower in the two intended parents which will open in the next day morning were protected by paper packet. In the next day morning both the flowers in two parental lines opened remaining inside the paper bag. The bags were removed and the pollens of the desired male parent in the crossing scheme 
were deposited over the stigma of the intended female parent within 7.30 a.m. After hybridization, the cross pollinated flowers were again bagged with fresh paper packet and kept for two more days. The fruits developed by hybridization were harvested at ripe stage when the ripe fruits split open exposing the matured and hard seeds covered with dark red aril. After removing the red aril from the seeds were washed thoroughly, dried in shade and then in sun. The dried seeds were stored in desiccators for further use.

\section{Evaluation of the parental genotypes and hybrids}

The 17 genotypes ( 7 parental inbred lines and 10 hybrids including one reciprocal) were evaluated during rainy-autumn season (seed sowing in mid-July) under side ventilated low cost poly house. The genotypes were grown in randomized block design with 3 replications keeping 5 plants in each replication in $60 \mathrm{x}$ 100 spacing to study the manifestation of different characters in them.

Three random plants per replication in each genotype were selected for recording the data on different characters.

\section{Manifestation of heterosis}

Heterosis over mid parent $(\mathrm{H})$ and Heterosis over better parent $(\mathrm{Hb})$ are measured as proportions of deviation of $\mathrm{F}_{1}$ values from mid parent and better parent respectively.

\section{Heterosis over the MP $(\mathrm{H})$}

It is commonly known as relative heterosis

and was calculated by - $\frac{\mathrm{F}_{1}-\mathrm{MP}}{\mathrm{MP}} \times 100$

Where, $\mathrm{F}_{1}$ and $\mathrm{MP}$ are the average performances of $\mathrm{F}_{1}$ and Mid parental values respectively.

\section{Heterosis over better parent (Hb)}

It is commonly known as Heterobeltiosis and

was calculated by - $\frac{F_{1}-B P}{B P}$

$$
\frac{\mathrm{F}_{1}-\mathrm{BP}}{\mathrm{BP}} \times 100
$$

Where, $\mathrm{F}_{1}$ and $\mathrm{BP}$ are the average performances of $\mathrm{F}_{1}$ and better parent respectively.

\section{Test of significance of heterosis}

Significant difference between the values in the Hybrids with respect to the Mid-parents and better parents (manifestation of heterosis) is determined following Falconer and Mackay (1996) as under:

$\mathrm{SE}(\mathrm{H})=\sqrt{\frac{3 \mathrm{Me}}{2 \mathrm{r}}}$

$\mathrm{SE}(\mathrm{Hb})=\sqrt{\frac{2 \mathrm{Me}}{\mathrm{r}}}$

Where, "Me" is the error mean square and "r" is the number of replication in the RBD analysis

Significance of $\mathrm{H}$ and $\mathrm{Hb}$ were determined by a t-test as follows:

$\mathrm{t}($ mid-parent $)=\mathrm{H} \div \mathrm{SE}(\mathrm{H})$

$\mathrm{t}($ better parent $)=\mathrm{Hb} \div \mathrm{SE}(\mathrm{Hb})$

The calculated ' $\mathrm{t}$ ' for both " $\mathrm{H}$ and $\mathrm{Hb}$ " is compared with the Table ' $t$ ' values at error degrees of freedom at 1 and $5 \%$ level of significance

If the calculated ' $t$ ' for the particular heterosisestimate $(\mathrm{H}$ or $\mathrm{Hb})$ is higher than the table ' $t$ ', the particular heterosis estimate is significant. 


\section{Results and Discussion}

Variation among the inbred lines and hybrids for different characters

Mean performance of the inbred lines and hybrids

Mean of the 14 different characters recorded in 17 genotypes (7 inbred lines and 10 hybrids) have been presented in Table 1 and 2 . The data for different characters viz., vine length $(\mathrm{m})$, branches per plant, days to first staminate flower, days to first pistillate flower, fruit length $(\mathrm{cm})$, fruit girth $(\mathrm{cm})$, fruit weight $(\mathrm{g})$, fruit number per plant, number of seeds per fruit, fruit yield per plant $(\mathrm{g})$, showed appreciable variation among the genotypes, showing the suitability of these genotypes for carrying out this study of variability for different characters.

\section{Analysis of heterosis for different characters}

Analysis of variance showed significant difference due to genotypes for all the characters for which manifestation of heterosis was calculated. This indicates presence of sufficient amount of variation for all the traits and selection will be effective in improving them. Heterosis is the superiority of $F_{1}$ over the mean of the parents or over the better parent or over the standard check (Hayes et al., 1956).

Significant better parent heterosis in desired direction is used for selection of best hybrids. Manifestations of heterosis for different characters have been discussed below.

Plant growth (Vine length and branches per plant)

Average vine length of the hybrids $(1.52 \mathrm{~m})$ was lesser than the parental mean $(1.65 \mathrm{~m})$ however, nine hybrids manifested significant positive heterosis ranging between $7.53 \%$ and $41.56 \%$ over the better parent. These results are in agreement with those of Choudhari and Kale (1991), Ranpise et al., (1992), Mishra et al., (1994), Laxuman et al., (2012) and Singh et al., (2013). Vine length ranged between between $1.31 \mathrm{~m}$ in BG-1346501 x Itki and $1.87 \mathrm{~m}$ in Meghna-2 x Murhu among the hybrids.

Average branches per plant of the hybrids (22.91) was higher than the parental mean (22.47) however, only two hybrids manifested significant positive heterosis ranging between $16.28 \%$ to $20.06 \%$ over the better parent. Number of branch per plant ranged between 19.40 in PusaVishesh x Meghna-2 and 27.64 in BG-1346501 x Special Boulder among the hybrids.

\section{Earliness}

In this investigation it was recorded (Table 3) that days to both staminate and pistillate flower were correlated positively and significantly $(\mathrm{r}=0.67)$. Hence, earliness could be judged from either days to staminate or pistillate flower in the vine. In bitter gourd, earliness is a useful character for realizing the potential economic yield in a short time. The characters like days to first male and female flower appeared and node number at which first male and female flower appeared are considered as criterion for earliness and in negative direction is desirable.

Average days to first staminate flower of the hybrids (38.11) was lesser than the parental mean (41.48) however, only five hybrids manifested significantly negative but very low level heterosis ranging between $-2.10 \%$ to $4.37 \%$ over the better parent. Days to first staminate flower ranged between 32.65 in Pusa Vishesh $x$ Meghna-2 and 49.81 in Meghna-2 x Murhu among the hybrids. 
Table.1 Mean of different plant and flowering characters in the inbreds and hybrids

\begin{tabular}{|c|c|c|c|c|}
\hline Genotype & vine length(m) & $\begin{array}{c}\text { No. of } \\
\text { branches/plant }\end{array}$ & $\begin{array}{l}\text { Days to first } \\
\text { staminate } \\
\text { flower }\end{array}$ & $\begin{array}{l}\text { Days to first } \\
\text { pistillate flower }\end{array}$ \\
\hline Kerala(W) & 1.55 & 19.83 & 34.27 & 48.59 \\
\hline Spl-B & 1.53 & 23.02 & 35.65 & 51.72 \\
\hline Meghna & 1.29 & 16.54 & 44.98 & 53.62 \\
\hline BG-1346501 & 1.93 & 22.33 & 35.30 & 41.67 \\
\hline Pusa vishesh & 1.22 & 21.05 & 34.14 & 49.94 \\
\hline Murhu & 3.20 & 31.01 & 61.75 & 54.59 \\
\hline$\overline{\text { Itki }}$ & 0.87 & 23.53 & 44.29 & 51.12 \\
\hline KeralaXMeghna & 1.67 & 23.06 & 44.95 & 53.95 \\
\hline MeghnaXBG-501 & 1.34 & 22.12 & 43.67 & 54.08 \\
\hline BG-501XSpl-B & 1.60 & 27.64 & 33.99 & 37.87 \\
\hline MeghnaXMurhu & 1.87 & 21.82 & 49.81 & 56.03 \\
\hline $\begin{array}{l}\text { BG-501XPusa } \\
\text { vishesh }\end{array}$ & 1.40 & 19.84 & 33.47 & 51.61 \\
\hline $\begin{array}{l}\text { Pusa } \\
\text { visheshXMeghna }\end{array}$ & 1.40 & 19.40 & 32.65 & 41.91 \\
\hline Spl-BXKerala & 1.47 & 22.56 & 33.11 & 38.94 \\
\hline BG-501XItki & 1.31 & 22.63 & 34.56 & 42.34 \\
\hline $\begin{array}{l}\text { Pusa visheshXBG- } \\
501\end{array}$ & 1.32 & 22.67 & 34.29 & 40.96 \\
\hline BG-501XMurhu & 1.83 & 27.39 & 40.67 & 44.61 \\
\hline $\mathbf{S E}(\mathbf{m})$ & 0.09 & 1.10 & 0.51 & 0.64 \\
\hline$\overline{\mathrm{SE}(d)}$ & 0.13 & 1.55 & 0.72 & 0.91 \\
\hline CD at $5 \%$ & 0.26 & 3.18 & 1.47 & 1.85 \\
\hline CD at $1 \%$ & 0.35 & 4.26 & 1.97 & 2.48 \\
\hline $\mathrm{CV}(\%)$ & 10.16 & 8.38 & 2.23 & 2.32 \\
\hline
\end{tabular}




\section{Int.J.Curr.Microbiol.App.Sci (2018) 7(10): 1376-1385}

Table. 2 Mean of different fruit and seed characters in the inbreds and hybrids

\begin{tabular}{|c|c|c|c|c|c|c|c|}
\hline Genotypes & $\begin{array}{l}\text { Fruit length } \\
(\mathrm{cm})\end{array}$ & $\begin{array}{l}\text { Fruit girth } \\
\text { (cm) }\end{array}$ & $\begin{array}{l}\text { Fruit weight } \\
\text { (g) }\end{array}$ & $\begin{array}{l}\text { Fruit } \\
\text { number/plant }\end{array}$ & $\begin{array}{c}\text { Fruit } \\
\text { yield/plant (g) }\end{array}$ & $\begin{array}{l}\text { Number of } \\
\text { Seed/fruit }\end{array}$ & $\begin{array}{l}\text { Weight of } 100 \\
\text { seeds }(\mathrm{g})\end{array}$ \\
\hline Kerala (W) & 14.45 & 17.09 & 54.66 & 8.34 & 417 & 16.48 & 26.64 \\
\hline Special Boulder (Spl-B) & 7.54 & 12.35 & 32.28 & 17.05 & 458.19 & 12.86 & 13.07 \\
\hline Meghna-2 & 12.82 & 15.32 & 62.11 & 14.25 & 753.45 & 20.19 & 22.21 \\
\hline BG-1346501 & 16.42 & 15.07 & 83.37 & 7.18 & 447.08 & 23.21 & 31.88 \\
\hline Pusavishesh & 15.02 & 17.31 & 65.13 & 8.46 & 442.07 & 17.93 & 25.20 \\
\hline Murhu & 4.42 & 6.41 & 14.32 & 17.42 & 234.78 & 10.60 & 11.39 \\
\hline Itki & 4.98 & 7.77 & 10.31 & 16.52 & 146.77 & 5.36 & 17.10 \\
\hline KeralaXMeghna-2 & 13.06 & 16.17 & 67.59 & 10.26 & 576.17 & 22.59 & 22.01 \\
\hline Meghna-2 XBG-1346501 & 14.24 & 14.96 & 101.71 & 14.89 & 1260.54 & 23.05 & 24.21 \\
\hline BG-1346501XSpl-B & 12.85 & 14.29 & 59.96 & 18.11 & 1050.62 & 18.59 & 23.27 \\
\hline Meghna-2 XMurhu & 8.94 & 12.08 & 45.52 & 16.27 & 634.38 & 21.21 & 20.59 \\
\hline BG-501XPusa vishesh & 17.80 & 16.07 & 112.84 & 9.13 & 867.84 & 20.26 & 23.88 \\
\hline PusavisheshXMeghna-2 & 13.92 & 15.71 & 72.64 & 8.64 & 584.81 & 18.89 & 22.39 \\
\hline Spl-BXKerala & 11.53 & 14.36 & 53.97 & 17.49 & 936.48 & 14.59 & 24.35 \\
\hline BG-1346501XItki & 12.99 & 14.15 & 52.17 & 15.12 & 703.90 & 15.09 & 17.90 \\
\hline Pusa visheshXBG-501 & 12.81 & 15.08 & 74.04 & 9.36 & 588.73 & 21.36 & 24.07 \\
\hline BG-1346501XMurhu & 10.46 & 14.23 & 56.93 & 12.51 & 659.34 & 17.33 & 18.21 \\
\hline $\mathbf{S E}(\mathbf{m})$ & 0.49 & 0.39 & 2.10 & 0.65 & 32.70 & 1.03 & 0.51 \\
\hline $\mathrm{SE}(\mathrm{d})$ & 0.69 & 0.55 & 2.98 & 0.92 & 46.24 & 1.46 & 0.73 \\
\hline CD at $5 \%$ & 1.42 & 1.13 & 6.10 & 1.88 & 94.63 & 2.99 & 1.50 \\
\hline CD at $1 \%$ & 1.90 & 1.51 & 8.16 & 2.52 & 126.62 & 4.09 & 2.07 \\
\hline CV (\%) & 7.07 & 4.82 & 6.08 & 8.67 & 8.94 & 10.17 & 4.14 \\
\hline
\end{tabular}




\section{Int.J.Curr.Microbiol.App.Sci (2018) 7(10): 1376-1385}

Table.3 Correlation among different characters of the genotypes (parental and hybrid lines)

\begin{tabular}{|c|c|c|c|c|c|c|c|c|c|c|c|c|c|c|c|c|c|c|c|c|c|}
\hline Character & VL & NBPP & DFSF & DFPF & DVCS & DVCP & $\mathrm{AT}$ & $\mathbf{A L}$ & SL & PV & PD & FL & FG & FW & FNPP & $\mathrm{TC}$ & BC & $\mathbf{A A}$ & NSPF & WS & FYPP \\
\hline VL & 1.00 & $0.65^{* *}$ & $0.64^{* *}$ & $0.18^{\mathrm{NS}}$ & $0.04^{\mathrm{NS}}$ & $-0.40^{\mathrm{NS}}$ & $-0.05^{\mathrm{NS}}$ & $-0.50^{*}$ & $-0.10^{\mathrm{NS}}$ & $-0.24^{\mathrm{NS}}$ & $0.26^{\mathrm{NS}}$ & $-0.35^{\mathrm{NS}}$ & $-0.45^{\mathrm{NS}}$ & $-0.27^{\mathrm{NS}}$ & $0.17^{\mathrm{NS}}$ & $-0.15^{\mathrm{NS}}$ & $-0.07^{\mathrm{NS}}$ & $0.25^{\mathrm{NS}}$ & $-0.04^{\mathrm{NS}}$ & $-0.33^{\mathrm{NS}}$ & $-0.23^{\mathrm{NS}}$ \\
\hline$\overline{\text { NBPP }}$ & & 1.00 & $0.43^{\mathrm{NS}}$ & $-0.12^{\mathrm{NS}}$ & $-0.01^{\mathrm{NS}}$ & $0.02^{\mathrm{NS}}$ & $0.15^{\mathrm{NS}}$ & $-0.39^{\mathrm{NS}}$ & $-0.37^{\mathrm{NS}}$ & $0.20^{\mathrm{NS}}$ & $0.04^{\mathrm{NS}}$ & $-0.56^{*}$ & $-0.61^{* *}$ & $-0.45^{\mathrm{NS}}$ & $0.47^{\mathrm{NS}}$ & $-0.34^{\mathrm{NS}}$ & $-0.24^{\mathrm{NS}}$ & $0.50^{*}$ & $-0.37^{\mathrm{NS}}$ & $-0.50^{*}$ & $-0.14^{\mathrm{NS}}$ \\
\hline DFSF & & & 1.00 & $0.67^{* *}$ & $0.20^{\mathrm{NS}}$ & $-0.18^{\mathrm{NS}}$ & $-0.39^{\mathrm{NS}}$ & $-0.54^{*}$ & $-0.17^{\mathrm{NS}}$ & $0.01^{\mathrm{NS}}$ & $-0.02^{\mathrm{NS}}$ & $-0.65^{* *}$ & $-0.69^{* *}$ & $-0.47^{\mathrm{NS}}$ & $0.42^{\mathrm{NS}}$ & $0.32^{\mathrm{NS}}$ & $0.38^{\mathrm{NS}}$ & $0.13^{\mathrm{NS}}$ & $-0.21^{\mathrm{NS}}$ & $-0.54^{*}$ & $-0.28^{\mathrm{NS}}$ \\
\hline DFPF & & & & 1.00 & $0.08^{\mathrm{NS}}$ & $-0.16^{\mathrm{NS}}$ & $-0.44^{\mathrm{NS}}$ & $-0.33^{\mathrm{NS}}$ & $0.04^{\mathrm{NS}}$ & $-0.02^{\mathrm{NS}}$ & $-0.24^{\mathrm{NS}}$ & $-0.30^{\mathrm{NS}}$ & $-0.27^{\mathrm{NS}}$ & $-0.14^{\mathrm{NS}}$ & $0.11^{\mathrm{NS}}$ & $0.36^{\mathrm{NS}}$ & $0.47^{\mathrm{NS}}$ & $-0.12^{\mathrm{NS}}$ & $-0.04^{\mathrm{NS}}$ & $-0.36^{\mathrm{NS}}$ & $-0.21^{\mathrm{NS}}$ \\
\hline$\overline{\text { DVCS }}$ & & & & & 1.00 & $-0.39^{\mathrm{NS}}$ & $-0.34^{\mathrm{NS}}$ & $-0.15^{\mathrm{NS}}$ & $0.05^{\mathrm{NS}}$ & $0.07^{\mathrm{NS}}$ & $0.12^{\mathrm{NS}}$ & $-0.10^{\mathrm{NS}}$ & $-0.08^{\mathrm{NS}}$ & $-0.05^{\mathrm{NS}}$ & $-0.06^{\mathrm{NS}}$ & $0.24^{\mathrm{NS}}$ & $0.29^{\mathrm{NS}}$ & $-0.08^{\mathrm{NS}}$ & $-0.09^{\mathrm{NS}}$ & $-0.17^{\mathrm{NS}}$ & $-0.07^{\mathrm{NS}}$ \\
\hline$\overline{D V C P}$ & & & & & & 1.00 & $-0.00^{\mathrm{NS}}$ & $-0.10^{\mathrm{NS}}$ & $-0.19^{\mathrm{NS}}$ & $0.61^{* *}$ & $-0.51^{*}$ & $-0.40^{\mathrm{NS}}$ & $-0.17^{\mathrm{NS}}$ & $-0.45^{\mathrm{NS}}$ & $0.41^{\mathrm{NS}}$ & $0.24^{\mathrm{NS}}$ & $0.23^{\mathrm{NS}}$ & $-0.00^{\mathrm{NS}}$ & $-0.45^{\mathrm{NS}}$ & $-0.27^{\mathrm{NS}}$ & $-0.08^{\mathrm{NS}}$ \\
\hline$\overline{\mathrm{AT}}$ & & & & & & & 1.00 & $0.42^{\mathrm{NS}}$ & $-0.49^{*}$ & $-0.26^{\mathrm{NS}}$ & $0.23^{\mathrm{NS}}$ & $0.43^{\mathrm{NS}}$ & $0.27^{\mathrm{NS}}$ & $0.39^{\mathrm{NS}}$ & $-0.36^{\mathrm{NS}}$ & $-0.58^{*}$ & $-0.64^{* *}$ & $-0.01^{\mathrm{NS}}$ & $0.29^{\mathrm{NS}}$ & $0.43^{\mathrm{NS}}$ & $0.11^{\mathrm{NS}}$ \\
\hline$\overline{\mathbf{A L}}$ & & & & & & & & 1.00 & $0.05^{\mathrm{NS}}$ & $-0.05^{\mathrm{NS}}$ & $0.37^{\mathrm{NS}}$ & $0.60^{* *}$ & $0.55^{*}$ & $0.61^{* *}$ & $-0.24^{\mathrm{NS}}$ & $-0.16^{\mathrm{NS}}$ & $-0.30^{\mathrm{NS}}$ & $0.04^{\mathrm{NS}}$ & $0.52^{*}$ & $0.44^{\mathrm{NS}}$ & $0.48^{\mathrm{NS}}$ \\
\hline SL & & & & & & & & & 1.00 & $-0.22^{\mathrm{NS}}$ & $-0.08^{\mathrm{NS}}$ & $0.10^{\mathrm{NS}}$ & $0.19^{\mathrm{NS}}$ & $0.21^{\mathrm{NS}}$ & $-0.05^{\mathrm{NS}}$ & $0.15^{\mathrm{NS}}$ & $0.09^{\mathrm{NS}}$ & $-0.11^{\mathrm{NS}}$ & $0.24^{\mathrm{NS}}$ & $0.16^{\mathrm{NS}}$ & $0.20^{\mathrm{NS}}$ \\
\hline$\overline{\mathbf{P V}}$ & & & & & & & & & & 1.00 & $-0.31^{\mathrm{NS}}$ & $-0.37^{\mathrm{NS}}$ & $-0.18^{\mathrm{NS}}$ & $-0.29^{\mathrm{NS}}$ & $0.57^{*}$ & $0.37^{\mathrm{NS}}$ & $0.45^{\mathrm{NS}}$ & $0.47^{\mathrm{NS}}$ & $-0.39^{\mathrm{NS}}$ & $-0.55^{*}$ & $0.21^{\mathrm{NS}}$ \\
\hline PD & & & & & & & & & & & 1.00 & $0.29^{\mathrm{NS}}$ & $0.06^{\mathrm{NS}}$ & $0.22^{\mathrm{NS}}$ & $-0.11^{\mathrm{NS}}$ & $-0.19^{\mathrm{NS}}$ & $-0.31^{\mathrm{NS}}$ & $0.32^{\mathrm{NS}}$ & $0.27^{\mathrm{NS}}$ & $0.21^{\mathrm{NS}}$ & $0.10^{\mathrm{NS}}$ \\
\hline$\overline{F L}$ & & & & & & & & & & & & 1.00 & $0.90^{* *}$ & $0.90^{* *}$ & $-0.70^{* *}$ & $-0.37^{\mathrm{NS}}$ & $-0.44^{\mathrm{NS}}$ & $-0.17^{\mathrm{NS}}$ & $0.74^{* *}$ & $0.82^{* *}$ & $0.48^{*}$ \\
\hline FG & & & & & & & & & & & & & 1.00 & $0.76^{* *}$ & $-0.66^{* *}$ & $-0.25^{\mathrm{NS}}$ & $-0.33^{\mathrm{NS}}$ & $-0.29^{\mathrm{NS}}$ & $0.71^{* *}$ & $0.73^{* *}$ & $0.49^{*}$ \\
\hline$\overline{F W}$ & & & & & & & & & & & & & & 1.00 & $-0.58^{*}$ & $-0.35^{\mathrm{NS}}$ & $-0.37^{\mathrm{NS}}$ & $0.01^{\mathrm{NS}}$ & $0.82^{* *}$ & $0.69^{* *}$ & $0.64^{* *}$ \\
\hline FNPP & & & & & & & & & & & & & & & 1.00 & $0.30^{\mathrm{NS}}$ & $0.31^{\mathrm{NS}}$ & $0.51^{*}$ & $-0.51^{*}$ & $-0.64^{* *}$ & $0.18^{\mathrm{NS}}$ \\
\hline TC & & & & & & & & & & & & & & & & 1.00 & $0.93^{* *}$ & $-0.06^{\mathrm{NS}}$ & $-0.19^{\mathrm{NS}}$ & $-0.36^{\mathrm{NS}}$ & $-0.16^{\mathrm{NS}}$ \\
\hline$\overline{B C}$ & & & & & & & & & & & & & & & & & 1.00 & $0.04^{\mathrm{NS}}$ & $-0.26^{\mathrm{NS}}$ & $-0.51^{*}$ & $-0.20^{\mathrm{NS}}$ \\
\hline$\overline{\mathbf{A A}}$ & & & & & & & & & & & & & & & & & & 1.00 & $-0.05^{\mathrm{NS}}$ & $-0.45^{\mathrm{NS}}$ & $0.39^{\mathrm{NS}}$ \\
\hline NSPF & & & & & & & & & & & & & & & & & & & 1.00 & $0.66^{* *}$ & $0.54^{*}$ \\
\hline$\overline{\text { WS }}$ & & & & & & & & & & & & & & & & & & & & 1.00 & $0.32^{\mathrm{NS}}$ \\
\hline
\end{tabular}

VL-Vine length; NBPP-Number of branches/plant; DFSF-Days to $1^{\text {st }}$ staminate flower; DFPF-Days to $1^{\text {st }}$ pistillate flower; DVCS-Days to visible corolla to anthesis (staminate) ; DVCP-Days to visible corolla to anthesis (Pistillate); AT-Anthesis time (am); AL-Anther length (mm); SL-Style length (mm); PV-Pollen viability (\%); PD-Pollen dia. $(\mu \mathrm{m})$; FL-Fruit length $(\mathrm{cm})$; FG-Fruit girth $(\mathrm{cm})$; FW-Fruit weight $(\mathrm{g})$; FNPP-Fruit number/plant; TC-Total chlorophyll $(\mathrm{mg} / 100 \mathrm{~g}$ fresh); BC-Beta-carotene (mg/100gfresh) AA-Ascorbic acid (mg/100g fresh); NSPF-Number of seed/fruit; WS-Weight of seed (100seeds); FYPP-Fruit yield per plant. 


\section{Int.J.Curr.Microbiol.App.Sci (2018) 7(10): 1376-1385}

Table.4 Manifestation of heterosis for different characters in the hybrids

\begin{tabular}{|c|c|c|c|c|c|c|c|c|c|c|c|c|c|c|c|c|c|c|}
\hline \multirow[t]{2}{*}{ Genotype } & \multicolumn{2}{|c|}{ Vine length } & \multicolumn{2}{|c|}{$\begin{array}{c}\text { No. of } \\
\text { branches/plant }\end{array}$} & \multicolumn{2}{|c|}{$\begin{array}{l}\text { days to first } \\
\text { staminate } \\
\text { flower }\end{array}$} & \multicolumn{2}{|c|}{$\begin{array}{c}\text { days to first } \\
\text { Pistillate flower }\end{array}$} & \multicolumn{2}{|c|}{$\begin{array}{c}\text { Fruit } \\
\text { length }(\mathrm{cm})\end{array}$} & \multicolumn{2}{|c|}{$\begin{array}{c}\text { Fruit } \\
\operatorname{girth}(\mathrm{cm})\end{array}$} & \multicolumn{2}{|c|}{$\begin{array}{c}\text { Fruit } \\
\text { weight(g) }\end{array}$} & \multicolumn{2}{|c|}{$\begin{array}{c}\text { Fruit } \\
\text { number/plant }\end{array}$} & \multicolumn{2}{|c|}{$\begin{array}{c}\text { Fruit } \\
\text { yield/plant(g) }\end{array}$} \\
\hline & MP & $\mathrm{BP}$ & MP & $\mathrm{BP}$ & MP & $\mathrm{BP}$ & MP & $\mathrm{BP}$ & MP & $\mathrm{BP}$ & MP & $\mathrm{BP}$ & MP & $\mathrm{BP}$ & MP & $\mathrm{BP}$ & MP & $\mathrm{BP}$ \\
\hline KeralaXMeghna & $17.35^{* *}$ & $7.53 * *$ & $26.82 * *$ & $16.28 * *$ & $13.43 * *$ & $31.14 * *$ & $5.57 * *$ & $11.02 * *$ & $-4.24 * *$ & $-9.66^{* *}$ & -0.23 & $-5.42 * *$ & $15.77 * *$ & $8.82 * *$ & $-8.13 * *$ & $-26.69 * *$ & $-13.33 * *$ & $-23.53 * *$ \\
\hline MeghnaXBG-501 & $-16.84 * *$ & $-30.56 * *$ & $-13.85 * *$ & -0.90 & $8.78 * *$ & $23.70 * *$ & $13.50 * *$ & $29.77 * *$ & $-2.57 * *$ & $13.25 * *$ & $-1.29 *$ & $-2.30 *$ & $39.82 * *$ & $21.99 * *$ & $40.55 * *$ & $6.36 * *$ & $109.99 * *$ & $67.30 * *$ \\
\hline BG-501XSpl-B & $-7.34 * *$ & $-16.94 * *$ & $21.90 * *$ & $20.06 * *$ & $-4.19 * *$ & $-3.71 * *$ & $-18.89 * *$ & $-9.11 * *$ & $7.23 * *$ & $21.74 * *$ & $4.51 * *$ & $-4.73 * *$ & 3.69 & $28.08 * *$ & $49.42 * *$ & $6.20 * *$ & $132.11 * *$ & $129.29 * *$ \\
\hline MeghnaXMurhu & $-16.75 * *$ & $-41.56 * *$ & $-8.21 * *$ & $-29.62 * *$ & $-6.65 * *$ & $10.73 * *$ & $3.56^{* *}$ & $4.50 * *$ & $3.79 * *$ & $30.21 * *$ & $11.21 * *$ & $21.12 * *$ & $19.11 * *$ & $26.71 * *$ & $3.55^{* *}$ & $-6.62 * *$ & $28.39 *$ & $-15.80 *$ \\
\hline $\begin{array}{l}\text { BG-501XPusa } \\
\text { vishesh }\end{array}$ & $-11.30 * *$ & $-27.46 * *$ & $-8.50 * *$ & $-11.11 * *$ & $-3.60 * *$ & $-1.97 * *$ & $12.67 * *$ & $23.84 * *$ & $13.23 * *$ & $8.40 * *$ & -0.55 & $-7.17 * *$ & $51.96 * *$ & $35.34 * *$ & $16.74 * *$ & $7.91 * *$ & $95.22 *$ & $94.11 *$ \\
\hline $\begin{array}{l}\text { PusavisheshXMe } \\
\text { ghna }\end{array}$ & $11.11 * *$ & $8.27 * *$ & $3.24 *$ & $-7.81 * *$ & $-17.47 * *$ & $-4.37 * *$ & $-19.05 * *$ & $-16.06 * * *$ & $0.02 *$ & $-7.30 * *$ & $-3.69 * *$ & $-9.24 * *$ & $14.17 * *$ & $11.53 * *$ & 23. & $-38.24 * *$ & -2.16 & -22.38 \\
\hline Spl-BXKerala & $-4.63 * *$ & $-5.34 * *$ & $5.29 * *$ & -1.99 & $-5.29 * *$ & $-3.38 * *$ & $-22.36 * *$ & $-19.87 * *$ & $4.82 * *$ & $20.22 * *$ & $7.17 * *$ & -0.62 & $24.16^{* *}$ & -1.26 & $37.76 * *$ & $2.58 * *$ & $81.07 * *$ & $62.54 * *$ \\
\hline BG-501XItki & $-6.52 * *$ & $-32.12 * *$ & -1.30 & $-3.82 * *$ & $-13.16 * *$ & $-2.10^{*}$ & $-8.73 * *$ & $1.60 * *$ & $21.42 * *$ & $20.84 * *$ & $24.30 * *$ & $-5.66 * *$ & $11.38 * *$ & $37.42 * *$ & $27.58 * *$ & $-8.47 * *$ & $137.06 * *$ & $57.44 * *$ \\
\hline $\begin{array}{l}\text { Pusa } \\
\text { visheshXBG-501 }\end{array}$ & $-15.93 * *$ & $-31.24 * *$ & $4.52 * *$ & 1.53 & $-1.23 *$ & 0.43 & $-10.57 * *$ & $-1.71 *$ & $18.49 * *$ & $21.96 * *$ & $-6.63 * *$ & $12.85^{* *}$ & -0.29 & $11.20 * *$ & $19.62 * *$ & $10.58 * *$ & $32.44 *$ & $31.68 *$ \\
\hline BG-501XMurhu & $-28.65 * *$ & $-42.81 * *$ & $2.71 *$ & $-11.65^{* *}$ & -16.18 & 15.21 & $-7.31 * *$ & $7.04 * *$ & $0.45^{*}$ & $36.25 * *$ & $32.91 * *$ & $-5.15 * *$ & $16.55^{* *}$ & $31.71 * *$ & $1.69 *$ & $-28.18 * *$ & $93.40 * *$ & $47.48 * *$ \\
\hline
\end{tabular}


Average days to first pistillate flower of the hybrids (46.23) was lesser than the parental mean (50.17) however, only three hybrids manifested significantly negative but very low level heterosis ranging between $-1.71 \%$ to $19.87 \%$ over the better parent. Days to first pistillate flower ranged between 38.94 in Special Boulder x Kerala White and 56.03 in Meghna-2 x Murhu among the hybrids. Laxuman et al., (2012) also emphasized the importance of earliness for realizing potential economic yield in less time. Negative heterosis for days to staminate or pistillate flower as recorded in the present investigation has found support from earlier findings of Singh et al., (2000) and Radharaniet al., (2014).

\section{Fruit number per plant}

Average number of fruits in the hybrids (13.18) was higher than the parental mean (12.74) however, only five hybrids manifested significantly positive but very low level heterosis ranging between $2.58 \%$ to $10.58 \%$ over the better parent. Similar results were obtained by Mishra et al., (1994) and Laxuman et al., (2012). Total fruit number per plant ranged 8.64 in PusaVishesh x Meghna-2 and 18.11 in BG-1346501 x Special Boulder among the hybrids. Number of fruits per plant, fruit length and fruit weight form the most important closely related productivity traits.

\section{Fruit weight (g)}

Average fruit weight in the hybrids $(69.73 \mathrm{~g})$ was much higher than the parental mean (46.02 g). For this reason (Table 4), 9 hybrids manifested significantly positive and considerably high level heterosis ranging between $8.82 \%$ and $37.42 \%$ over the better parent.

Average fruit weight ranged between 10.31 gin Itki and $83.37 \mathrm{~g}$ inBG-1346501 among the parental lines and between $45.52 \mathrm{~g}$ in Meghna-2 $\mathrm{x}$ Murhu and 112.84 $\mathrm{g}$ in BG-1346501 $\mathrm{x}$ PusaVishesh among the hybrids.

\section{Fruit length $(\mathrm{cm})$}

Average fruit length in the hybrids $(12.86 \mathrm{~cm})$ was higher than the parental mean $(10.81 \mathrm{~cm})$. For this reason (Table 4), 8 hybrids manifested significantly positive and considerably high level heterosis ranging between $8.40 \%$ to 36.25 $\%$ over the better parent. Manifestation of heterosis for fruit length as recorded in this investigation was in agreement with those of Ranpise et al., (1992), Mishra et al., (1994) and Radharani et al., (2014).

Fruit length ranged between $4.98 \mathrm{~cm}$ in Itki and $16.42 \mathrm{~cm}$ in BG-1346501 among the parental lines and between $8.94 \mathrm{~cm}$ in Meghna-2 $\mathrm{x}$ Murhu and $17.80 \mathrm{~cm}$ in BG-1346501 $\mathrm{x}$ PusaVishesh among the hybrids.

\section{Fruit girth (cm)}

Average fruit girth in the hybrids $(14.71 \mathrm{~cm})$ was slightly higher than the parental mean $(13.04 \mathrm{~cm})$. However, only two hybrids manifested significantly positive and considerably high level heterosis ranging between $12.85 \%$ to $21.12 \%$ over the better parent. Fruit girth ranged between $12.08 \mathrm{~cm}$ in Meghna-2 x Murhu and $16.17 \mathrm{~cm}$ in Kerala White $x$ Meghna- 2 among the hybrids.

\section{Fruit yield per plant (g)}

Fruit yield per plant is the ultimate and the most important trait. Average fruit yield per plant in the hybrids (786.28 g) was markedly higher than the parental mean (414.24). For this reason, 7 hybrids manifested significantly positive and considerably high level positive heterosis ranging between $31.68 \%$ and 129.29 $\%$ over the better parent. Fruit yield per plant ranged between $588.73 \mathrm{~g}$ in PusaVishesh x BG1346501 and $1260.54 \mathrm{~g}$ in Meghna-2 x BG1346501 among the hybrids. Fruit yield per plant was greatly influenced by number of fruits; average fruit weight, fruit length and fruit girth and for these characters expression of heterosis in positive direction are desirable. 


\section{Identification of best hybrids}

Wide variation could be recorded among the parental genotypes as well as hybrids for most of the characters under study. Presence of dominance and complementary type of gene action for yield indicates that heterosis breeding would be more advantageous to get higher fruit yield in bitter gourd (Singh et al., 2013).

In terms of per se performance, and heterobeltosis, two hybrids, Meghna-2 x BG1346501 and BG-1346501 x Special Boulder were found superior. It is therefore, suggested that these promising parents and crosses may be exploited for further amelioration of yield and yield components in bitter gourd. The selected crosses can be directly utilized as promising hybrids.

\section{References}

Chaudhari, S. M. and Kale, P. N. 1991a. Combining ability studies in bitter gourd (Momordica charantia L.). South Indian Hort. 40(6): 313-315.

Hayes, H. K., Immer, F. F. and Smith, D. C. 1956. Methods of Plant Breeding. McGraw Hill Book Publishing Company, Inc., New Delhi.

Jeffrey C (1980). A review of the Cucurbitaceae. Botanical Journal of the Linnean Society, 81 233-247

Lawande, K. E. and Patil, A. V. 1990b. Studies on combining ability and gene action in bitter gourd. J. Maharashtra Agril. Univ. 19(1): 24-28.

Laxuman, S. A. Patil, P. M. Salimath, P. R. Dharmatti, A. S. Byadgi and Nirmalayenagi 2012. Heterosis and combining ability analysis for productivity traits in bitter gourd (Momordica charantia L.). Karnataka J. Agric. Sci. 25(1): 9-13.

Mishra, H. N., Mishra, R. S., Mishra, S. N. and Parhi, G. 1994. Heterosis and combining ability in bittergourd (Momordica charantia). Indian J. Agricultural Science. 64(5): 310-313

Okabe, H., Miyahara, Y. and Yamauchi, T. (1982). Studies on the constituents of Momordica charantia L. III. Chemical \& Pharmaceutical Bulletin, 30 3977-3986.

Radha Rani, K., Raju, C.H. and Reddy, K. R. (2014). A study on heterosis for yield and earliness in bitter gourd. Indian J. Sci. Res. and Tech. 2014 2(3):89-97

Ranpise, S. A., Kale, P. N., Desale, G. V. and Desai, U. T. 1992. Heterosis in bitter gourd (Momordica charantia L.). South Indian Hort. 40(6): 313-315.

Riggs, T. J. 1988. Breeding $\mathrm{F}_{1}$ hybrid varieties of vegetables. Journal of Horticultural Sciences. 63:362-369.

Singh A K, Pandey U B and M Singh 2000 Studies on heterosis in bitter gourd (Momordica charantia L.). Vegetable Science 27(2): 158-161.

Singh, A. K., Pan, R.S. and Bhavana, P. (2013). Heterosis and combining ability in bitter gourd (Momordica charantia L.). The Bioscan, 8: 1533-1536

Sirohi, P. S. and Choudhary, B. 1978. Heterosis in bitter gourd (Momordica charantia L.). Veg. Sci. 4(2): 107-115.

Welthinda, J., Karunanayake, E.M., Sheriff MH \& Jayasinghe KS (1986). Effect of Momordica charantia on the glucose tolerance in maturity onset diabetes. Journal of Ethnopharmacology, 17 277282.

\section{How to cite this article:}

Robindro Singh, P., ChandanKarak, Priyadarshani P. Mohapatra, B. Ashok Kumar and Hazra, P. 2018. Manifestation of Heterosis in Bittergourd. Int.J.Curr.Microbiol.App.Sci. 7(10): 1376-1385. doi: https://doi.org/10.20546/ijcmas.2018.710.153 